\title{
Ubiquitin-conjugating enzyme involved in the immune response caused by pathogens invasion
}

\author{
Liang Chen ${ }^{1,2}$, Chen Cheng ${ }^{2}$, Chunxia Zhang ${ }^{2}$, Qin Yao $^{2}$, Ermi Zhao ${ }^{1^{*}}$ \\ ${ }^{1}$ Key Laboratory of Bio-Resources and Eco-Environment, College of Life Sciences, Sichuan University, Chengdu, China; \\ *Corresponding Author: Ermizhao@gmail.com \\ ${ }^{2}$ Institute of Life Sciences, Jiangsu University, Zhenjiang, China
}

Received 27 May 2013; revised 25 June 2013; accepted 9 July 2013

Copyright (c) 2013 Liang Chen et al. This is an open access article distributed under the Creative Commons Attribution License, which permits unrestricted use, distribution, and reproduction in any medium, provided the original work is properly cited.

\begin{abstract}
Ubiquitin-proteasome pathway (UPP) is a significant way of protein degradation and modification in eukaryotic cell and involved in a complex series of intracellular processes. As a key component in UPP, ubiquitin-conjugating enzyme (E2) plays an extremely important role in ubiquitin (Ub) transferring and substrate specific recognition. Abundant evidences have proved that UPP is involved in cells immune reaction caused by pathogens and the attendance of E2 has a significant effect on host cells and pathogen. This article presents an overview of the current research on E2s that is involved in immune response caused by viruses and bacteria.
\end{abstract}

Keywords: Ubiquitin-Conjugating Enzyme; Ubiquitin-Proteasome Pathway; Pathogen; Immune Response

\section{INTRODUCTION}

As a pathway of protein degradation and posttranslational modification [1], ubiquitin-proteasome pathway (UPP) is responsible for the degradation of $80 \%-90 \%$ bulk protein in eukaryotic cell [2]. UPP is involved in a complex series of intracellular processes, such as gene expression regulation [3], cell cycle regulation, DNA damage repair, immune response [4], cell apoptosis [5] and so forth. To prevent cells from undergoing proteotoxic stress, UPP controls the levels of cellular proteome by regulating the complex series of intracellular processes [6]. UPP is a series of complex enzymatic cascade reaction and composed of ubiquitin-activating enzyme (E1), ubiquitin-conjugating enzyme (E2) and ubiquitin-ligating enzyme (E3) [7]. With the help of the three enzymes and adenosine triphosphate (ATP), UPP finishes ubiquitinating the substrate proteins. As the second enzyme of UPP, E2 is highly conserved in different species. E2 plays an extremely important role in ubiquitin (Ub) transferring and substrate specific recognition. In recent years, E3 and viral Ub become the focus of attention. There has recently been great progress in the functions of E3s and viral Ub gene. However, up to now, not much research has been performed on the functions of E2s in cells except the transferring of Ub. More and more researchers prove that UPP is involved in cellular immune reactions caused by pathogens. Interestingly, E2s have always been detected in the immune reactions. But the functions of E2s in immune reactions caused by pathogens are still unclear. This review presents an overview of the current researches on different E2s and the immune responses of cells caused by viruses and bacteria. The purpose of this review is to enhance understanding of the functions of E2s when cells suffer from the invasion of pathogens.

\section{UBIQUITIN-PROTEASOME PATHWAY}

UPP is a main way of protein degradation in eukaryotic cells. Ubiquitination of the target protein comprises three steps. Firstly, ATP-dependent E1 activated Ub by C-terminal adenylation, followed by formation of a high-energy thioester bond. Secondly, activated Ub is then transferred from E1 to the active-site cysteine of E2 preserving the thioester bond. Third, E3 promote transfer of Ub from E2 to the target protein [8-10]. At last, the targeted proteins are transported to proteasome for degradation (Figure 1) [11]. Actually, Ub is a kind of special protein with a very long life span and itself cannot be degraded. Polyubiquitin chain can be dissociated for target proteins with the help of deubiquitinating enzymes (DUBs). The dissociated Ubs can participate in targeting next substrate protein. In previous studies, the connection between the ubiquitination and the activation of the 


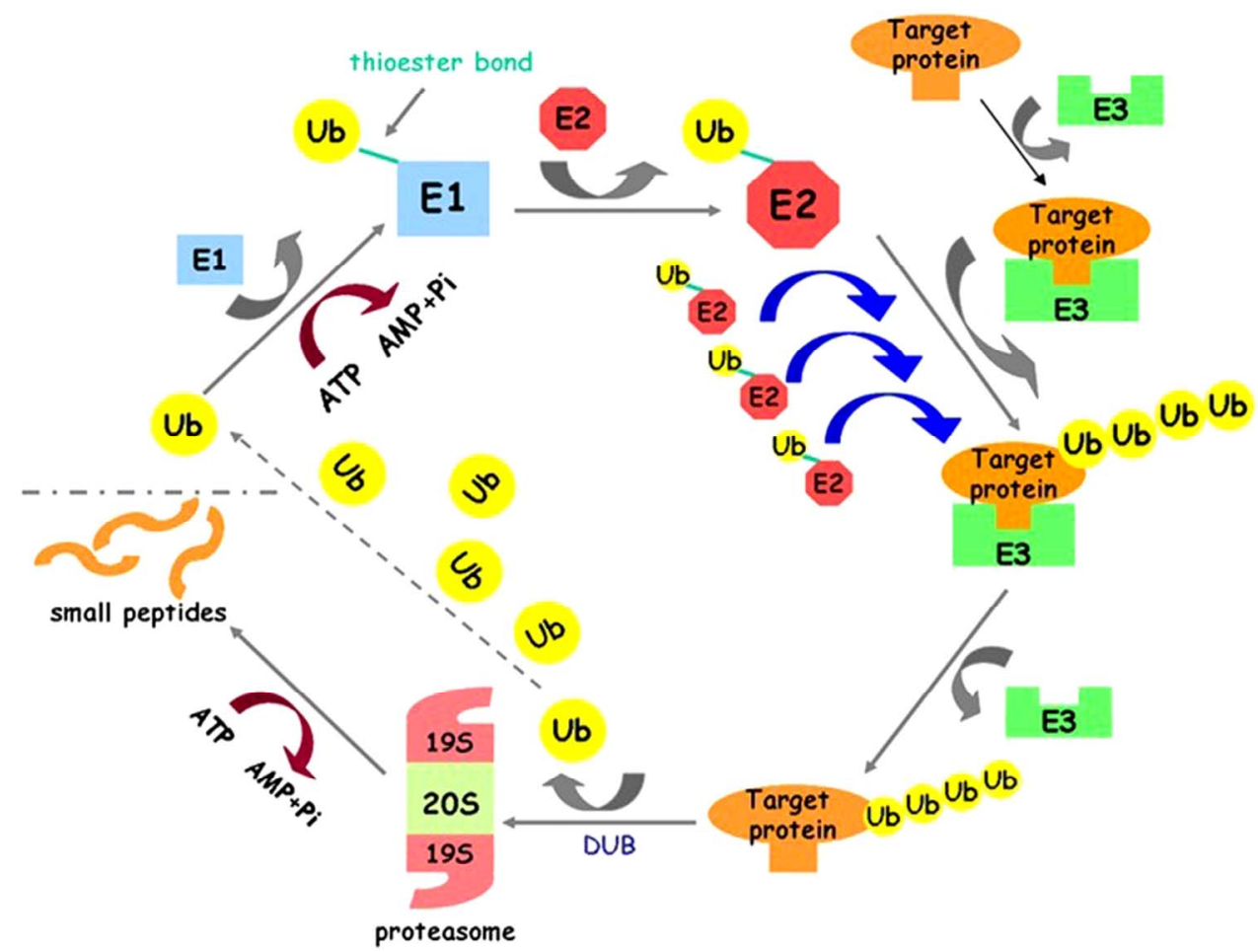

Figure 1. Ubiquitin-proteasome pathway (UPP). ATP-dependent E1 activated Ub by C-terminal adenylation, followed by formation of a high-energy thioester bond; activated Ub is then transferred from E1 to the active-site cysteine of E2 preserving the thioester bond; E3 promote transfer of Ub from E2 to the target protein. Polyubiquitin chain can be dissociated for target proteins with the help of deubiquitinating enzymes (DUBs). The dissociated Ubs can participate in targeting next substrate protein.

genes, which are involved in the cellular immune responses, has been identified. To control the activity of some key factors regarding to immune responses, cells are likely to up-regulate the expression level of genes involved in progress of protein modification, for example the cytokine TNF- $\alpha$ [12].

\section{THE CHARACTERISTICS AND CLASSES OF E2}

Eukaryotic genomes encode large families of E2s and eukaryotic E2 originates from the Guillardia theta nucleomorph about 2500 million years ago [2]. E2 is mainly vertically inherited through eukaryotic evolution [13]. In Guillardia theta nucleomorph, a lot of genes may have been lost during the process of evolution, and the genes only necessary for survival are retained. However, three E2 genes have been identified among 632 reference sequences [14]. Up to now, 17 kinds of E2s have been found in yeast and 13 in Arabidopsis thaliana [15]. Human genome encodes 60 - 70 E2s. The diversification of E2s is quite essential to the specificity of substrates. E2s all have a conservative UBC domain composed of 150 amino acids and within the UBC domain E2s possess a specific active site cysteine residue. The structure of E2 is comprised of an $\mathrm{N}$-termianal $\alpha$-helix, three C-termianal $\alpha$-helix and a four-stranded antiparallel $\beta$-sheet [16]. Most of the E2s are proteins with small molecular weight [17]. Based on structural analysis, E2s could be divided into four groups: Class I only consists of the conserved UBC domain [18]; Class II has additional C-terminal extensions and Class III has additional $\mathrm{N}$-terminal extensions [19,20]; Class IV possesses both $\mathrm{N}$ and C-terminal extensions [21]. Different classes of E2s are very meaningful to different target proteins of UPP.

\section{E2S AND CELLULAR IMMUNE RESPONSES}

Whether bacterium or virus, the invasion of pathogens can give rise to a series of cellular immune responses. UPP is the chief pathway of cellular protein hydrolysis. Nowadays, many researches are focused on the changes of enzymes involved in UPP when the pathogens invade host cells, in order to enhance understanding of the activities of pathogens in cells. E2, a key enzyme of UPP, can directly or indirectly conjugate Ub to bulk protein. Recently, it has been identified in some studies that E2 can interact with the proteins of pathogens. The research results not only do provide evidences for the interaction 
between the host proteins and pathogens, but lay the foundation for the further functions study of E2s. At present, the researches of E2s are largely focused on several model organisms, such as yeast, Arabidopsis thaliana and mouse. Furthermore, some E2s researchers place emphasis on the aspect of antitumor. Using DNA microarrays, an ubiquitin-conjugating enzyme E2C gene (UBE2C) was found to be the most highly overexpressed gene in both the primary tumors and the liver metastases [22]. Current studies have shown the increase of E2 expression levels challenged to some pathogens, such as bacteria and viruses. In the review, we sum the research results about interaction between E2s and pathogens recently and discuss the functions of E2s in cellular immune responses caused by viruses and bacteria.

\subsection{Immune Response Caused by Viruses}

Virus is a kind of highly parasitic microorganism. Besides, these microorganisms cannot survive and they must parasitize some organisms. By inhibiting the protein synthesis and metabolism or degradation of host proteins, virus plunders and exploits substrate for the proliferation of progeny virus. However, the invasion of virus can lead to a series of immune responses in cell. As a significant pathway of protein degradation, UPP is always utilized by some viruses. Some key enzymes of UPP are usually targeted by virus, for example, E2s. By interacting with the E2s, virus modify some cellular proteins, allowing them to escape antiviral defense system.

\subsubsection{Plant Viruses}

On the aspect of ubiquitination, most studied plants is Arabidopsis thaliana. In plant, there are approximately $5 \%$ total protein coded from 1400 genes that involved in UPP. What's more, 37 of those genes code E2s while 8 code E2-like proteins [23]. Now research slows that Cotton leaf curl Multan virus (CLCuMV), one of Geminiviruses, can encodes a single gene $\beta \mathrm{C} 1 . \beta \mathrm{C} 1$ is a kind of pathogenicity protein and important for typical symptom expression [24,25]. It has been reported that the DNA- $\beta$-specific symptoms of the host was induced by the interaction between $\beta \mathrm{C} 1$ and the tomato $\mathrm{E} 2$ (S1UBC3) [26]. In order to modify the host UPP, $\beta \mathrm{C} 1$ bind to S1UBC3 on the mytistoylation-like motif. The result shows that the E2 is a target of virus. With the help of the target protein, virus can succeed in escaping host defenses or exploiting host biological pathway.

\subsubsection{Animal Viruses}

The study on the interaction between animal viruses and the E2s is mainly focused on mammal in recent years. Researchers try to clarify the way in which the viruses plunder and exploit substrates for the prolifera- tion in host cells by investigating the interaction between host UPP and viruses. As the etiological agent of adult T-cell leukemia, Human T-cell leukemia virus type 1 (HTLV-1) encodes Tax which is a kind of regulatory protein [27]. It has been proved that Ubc13 is important to the ubiquitination of Tax. The ubiquitination of Tax oncoprotein can regulate the mechanism which promotes the activation of NF- $\kappa \mathrm{B}$ [28]. And then the activation of NF- $\kappa \mathrm{B}$ is able to facilitate transformation of T cells [29]. $\mathrm{T}$ cell transformation is beneficial to the virus itself. But the transformation is disadvantageous to the host cells. This situation has also been reported in the process of HIV infected cells. Tsg101, a kind of E2 variant protein, has been shown special interaction with the p6 region of HIV-1 Gag protein in mammalian cells. The interaction is relevant to the life cycle of virus [30]. The research results are similar to the discoveries of plants. Based on the current study results, some E2s are beneficial to viruses survival and proliferation during the viruses invasion. In other words viruses are good at making use of host enzymes. But not all the E2s can be exploited by the virus. A kind of Bombyx mori E2, involved in antivirus against nucleopolyhedrovirus, has been found by fluorescent differential display in our laboratory (data not published). The expression level of the E2 in the resistance strain was higher than the susceptible strain (data not published). These results imply various E2s possess different functions. It may be one of the reasons in biodiversity.

\subsection{Immune Responses Caused by Bacteria}

The bacterium is a class of etiological agent and the bacterial invasion can result in a series of cellular immune reactions. That E2s can interact with bacteria and help bacteria to invade host cells has also been reported in current studies. UBE2 gene of E2 overexpressed when Concholepas concholepas suffer from Vibrio anguillarum [31]. Besides, Shigella flexneri is responsible for shigellosis of humans. By targeting E2 of the host cell, Shigella flexneri effector OspG interferes with immune responses [32]. Interestingly, ectopic expression of the E2 from wild rice OgUBC1 in Arabidopsis thaliana confers resistance against Botrytis cinerea on the OgUBC1expressing plants [33]. Apparently, OgUBC1 is involved in the ubiquitination process of cellular immune response against Botrytis cinerea in plants. Taken together, the functions of E2s in cellular immune responses caused by bacteria are dissimilar. The diversification of the functions of E2s may be decided by the various structures of E2s.

\section{CONCLUSION}

In this review, we focused on showing the different 
functions of E2s in cellular immune responses caused by viruses and bacteria. As a key component of UPP, E2 is crucial for the protein degradation and modification. Previous studies have reported that pathogens interact with cellular immune responses by targeting host E2s. Successful viruses or bacteria have evolved superior strategies to escape host defenses or exploit host biological pathways. Some kinds of E2s, to a certain extent, assist viruses or bacteria to survive and proliferate in host cells. However, the E2s perform distinct functions in cellular response. Some E2s are likely to be concerned with resistance against pathogens. By enhancing some key genes related to immune response activity, some E2s can interfere with the survival and proliferation of pathogens. Therefore, the functions of E2s family in cell are not uniform. The difference of E2s makes the research of E2 family become difficult. However, with the tireless efforts, we have obtained a great many achievements on E2s researches. The researches on E2s not only provide us the functions of E2s family, but also help us to clarify the association between pathogens and host UPP. What's more, the association will assist us in understanding the exploitation process of metabolic substrates actualized by pathogens and help us find some potential targets to cure certain diseases.

\section{ACKNOWLEDGEMENTS}

This work was supported by Postdoctoral daily finance of Jiangsu University (No. 1143002139), and the National Basic Research Program (973) of China (No. 2012CB4604).

\section{REFERENCES}

[1] Ciechanover, A., Everett, R.D., Orr, A., et al. (1998) The ubiquitin-proteasome pathway: On protein death and cell life FREE. The EMBO Journal, 17, 7151-7160. doi:10.1093/emboj/17.24.7151

[2] Ying, M., Zhan, Z., Wang, W., et al. (2009) Origin and evolution of ubiquitin-conjugating enzymes from Guillardia theta nucleomorph to hominoid. Gene, 447, 72-85. doi:10.1016/j.gene.2009.07.021

[3] Yao, T. and Ndoja, A. (2012) Regulation of gene expression by the ubiquitin-proteasome system. Seminars in Cell \& Developmental Biology, 23, 523-529. doi:10.1016/j.semcdb.2012.02.006

[4] Gray, W.M. and Estelle, M. (2000) Function of the ubiquitin-proteasome pathway in auxin response. Trends in Biochemical Sciences, 25, 133-138. doi:10.1016/S0968-0004(00)01544-9

[5] Friedman, J. and Xue, D. (2004) To live or die by the sword: The regulation of apoptosis by the proteasome. Developmental Cell, 6, 460-461. doi:10.1016/S1534-5807(04)00104-2

[6] Lyupina, Y.V., Abaturova, S.B., Erokhov, P.A., et al. (2012) Proteotoxic stress induced by Autographa califor- nica nucleopolyhedrovirus infection of Spodoptera frugiperda Sf9 cells. Virology, 436, 49-58.

doi:10.1016/j.virol.2012.10.018

[7] Vierstra, R.D. (2003) The ubiquitin/26S proteasome pathway, the complex last chapter in the life of many plant proteins. Trends in Plant Science, 8, 135-142. doi:10.1016/S1360-1385(03)00014-1

[8] Ciechanover, A., Orian, A. and Schwartz, A.L. (2000) The ubiquitin-mediated proteolytic pathway: Mode of action and clinical implications. Journal of Cellular Biochemistry, 77, 40-51. doi:10.1002/(SICI)1097-4644(2000)77:34+<40::AID-JC B9>3.0.CO;2-6

[9] Zhao, C., Beaudenon, S.L., Kelley, M.L., et al. (2004) The UbcH8 ubiquitin E2 enzyme is also the E2 enzyme for ISG15, an IFN- $\alpha / \beta$-induced ubiquitin-like protein. Proceedings of the National Academy of Sciences of the United States of America, 101, 7578-7582. doi:10.1073/pnas.0402528101

[10] Kim, H.T., Kim, K.P., Lledias, F., et al. (2007) Certain pairs of ubiquitin-conjugating enzymes (E2s) and ubiquitin-protein ligases (E3s) synthesize nondegradable forked ubiquitin chains containing all possible isopeptide linkages. Journal of Biological Chemistry, 282, 17375-17386. doi:10.1074/jbc.M609659200

[11] Cheng, C., Yao, Q. and Chen. K. (in press) Functions regarding conjugating enzyme that participate in process of protein modification involved in immune response. Journal of Biology.

[12] Li, Y., Lecker, S.H., Chen, Y., et al. (2003) TNF- $\alpha$ increases ubiquitin-conjugating activity in skeletal muscle by up-regulating UbcH2/E220k. The FASEB Journal, 17, 1048-1057. doi:10.1096/fj.02-0759com

[13] Lespinet, O., Wolf, Y.I., Koonin, E.V., et al. (2002) The role of lineage-specific gene family expansion in the evolution of eukaryotes. Genome Research, 12, 1048-1059. doi:10.1101/gr.174302

[14] Douglas, S., Zauner, S., Fraunholz, M., et al. (2001) The highly reduced genome of an enslaved algal nucleus. $\mathrm{Na}$ ture, 410, 1091-1096. doi:10.1038/35074092

[15] Hegde, A.N. and Upadhya, S.C. (2011) Role of ubiquitinproteasome-mediated proteolysis in nervous system disease. Biochimica et Biophysica Acta (BBA)-Gene Regulatory Mechanisms, 1809, 128-140. doi:10.1016/j.bbagrm.2010.07.006

[16] Houben, K., Dominguez, C., van Schaik, F., et al. (2004) Solution structure of the ubiquitin-conjugating enzyme UbcH5B. Journal of Molecular Biology, 344, 513-526. doi:10.1016/j.jmb.2004.09.054

[17] van Wijk, S.J. and Timmers, H.M. (2010) The family of ubiquitin-conjugating enzymes (E2s): Deciding between life and death of proteins. The FASEB Journal, 24, 981993. doi:10.1096/fj.09-136259

[18] Zhang, X., Jenkins, J.N., Callahan, F.E., et al. (2003) Molecular cloning, differential expression, and functional characterization of a family of class I ubiquitin-conjugating enzyme (E2) genes in cotton ( Gossypium). Biochimica et Biophysica Acta (BBA)-Gene Structure and Expression, 1625, 269-279. doi:10.1016/S0167-4781(02)00623-1 
[19] Sun, B., Jeyaseelan, K., Chung, M., et al. (1997) Cloning, characterization and expression of a cDNA clone encoding rabbit ubiquitin-conjugating enzyme, E2 $2_{32 \mathrm{k}}$. Biochimica et Biophysica Acta (BBA)-Gene Structure and Expression, 1351, 231-238. doi:10.1016/S0167-4781(96)00209-6

[20] Müller, U., Grams, A., Martinez-Noel, G., et al. (1998) Structure of the gene encoding the ubiquitin-conjugating enzyme Ubcm4, characterization of its promoter, and chromosomal location. Gene, 224, 109-116. doi:10.1016/S0378-1119(98)00515-0

[21] Miura, T., Klaus, W., Gsell, B., et al. (1999) Characterization of the binding interface between ubiquitin and class I human ubiquitin-conjugating enzyme $2 \mathrm{~b}$ by multidimensional heteronuclear NMR spectroscopy in solution. Journal of Molecular Biology, 290, 213-228. doi:10.1006/jmbi.1999.2859

[22] Takahashi, Y., Ishii, Y., Nishida, Y., et al. (2006) Detection of aberrations of ubiquitin-conjugating enzyme E2C gene $(U B E 2 C)$ in advanced colon cancer with liver metastases by DNA microarray and two-color FISH. Cancer Genetics and Cytogenetics, 168, 30-35. doi:10.1016/j.cancergencyto.2005.12.011

[23] Smalle, J. and Vierstra, R.D. (2004) The ubiquitin 26S proteasome proteolytic pathway. Annual Review of Plant Biology, 55, 555-590. doi:10.1146/annurev.arplant.55.031903.141801

[24] Cui, X., Tao, X., Xie, Y., et al. (2004) A DNA $\beta$ associated with Tomato yellow leaf curl China virus is required for symptom induction. Journal of Virology, 78, 13966-13974.

[25] Qian, Y. and Zhou, X. (2005) Pathogenicity and stability of a truncated DNA $\beta$ associated with Tomato yellow leaf curl China virus. Virus Research, 109, 159-163. doi:10.1016/j.virusres.2004.11.017

[26] Eini, O., Dogra, S., Selth, L.A., et al. (2009) Interaction with a host ubiquitin-conjugating enzyme is required for the pathogenicity of a geminiviral DNA $\beta$ satellite. Molecular Plant-Microbe Interactions, 22, 737-746.

\section{doi:10.1094/MPMI-22-6-0737}

[27] Yao, J. and Wigdahl, B. (2000) Human T cell lymphotropic virus type I genomic expression and impact on intracellular signaling pathways during neurodegenerative disease and leukemia. Front Bioscience, 5, D138-D168. doi:10.2741/Yao

[28] Shembade, N., Harhaj, N.S., Yamamoto, M., et al. (2007) The human T-cell leukemia virus type 1 Tax oncoprotein requires the ubiquitin-conjugating enzyme Ubc13 for NF$\kappa \mathrm{B}$ activation. Journal of Virology, 81, 13735-13742. doi:10.1128/JVI.01790-07

[29] Harhaj, E.W. and Harhaj, N.S. (2005) Mechanisms of persistent NF- $\kappa$ B activation by HTLV-I tax. IUBMB Life, 57, 83-91. doi:10.1080/15216540500078715

[30] VerPlank, L., Bouamr, F., LaGrassa, T.J., et al. (2001) Tsg101, a homologue of ubiquitin-conjugating (E2) enzymes, binds the L domain in HIV type 1 Pr55Gag. Proceedings of the National Academy of Sciences, 98, 77247729. doi:10.1073/pnas.131059198

[31] Núñez-Acuña, G., Aguilar-Espinoza, A., Chávez-Mardones, J., et al. (2012) Ubiquitin-conjugating enzyme E2like gene associated to pathogen response in Concholepas concholepas: SNP identification and transcription expression. Fish \& Shellfish Immunology, 33, 1065-1068. doi:10.1016/j.fsi.2012.08.024

[32] Kim, D.W., Lenzen, G., Page, A.-L., et al. (2005) The Shigella flexneri effector OspG interferes with innate immune responses by targeting ubiquitin-conjugating enzymes. Proceedings of the National Academy of Sciences of the United States of America, 102, 14046-14051. doi:10.1073/pnas.0504466102

[33] Jeon, E.H., Pak, J.H., Kim, M.J., et al. (2012) Ectopic expression of ubiquitin-conjugating enzyme gene from wild rice, $O g U B C 1$, confers resistance against UV-B radiation and Botrytis infection in Arabidopsis thaliana. Biochemical and Biophysical Research Communications, 427, 309-314. 